\title{
The microRNA miR-124 suppresses seizure activity and regulates CREB1 activity
}

\author{
WEI WANG ${ }^{1}$, XUEFENG WANG ${ }^{1,2},{\text { LANG } \mathrm{CHEN}^{1} \text {, YUJIAO ZHANG }}^{1}, \mathrm{ZUCAI}^{\mathrm{X}} \mathrm{ZU}^{1}$, \\ JING LIU ${ }^{1}$, GUOHUI JIANG ${ }^{1}$, JIE LI ${ }^{1}$, XIAOGANG ZHANG ${ }^{1}$, KEWEI WANG ${ }^{3}$, \\ JINGHUI WANG ${ }^{4}$, GUOJUN CHEN ${ }^{1}$, JING LUO $^{1 *}$ \\ ${ }^{1}$ Department of Neurology, Chongqing Key Laboratory of Neurology, The First Affiliated Hospital of Chongqing \\ Medical University, 1 Youyi Road, Chongqing 400016, China, ${ }^{2}$ Center of Epilepsy, Beijing Institute for Brain \\ Disorders, Beijing 100101, China, ${ }^{3}$ Peking University School of Medicine, 38 Xueyuan Road, Beijing 100091, \\ China, and ${ }^{4}$ The Institute of Biophysics, Chinese Academy of Sciences, 15 Datun Road, Beijing 100101, China
}

miR-124, a brain-specific microRNA, was originally considered as a key regulator in neuronal differentiation and the development of the nervous system. Here we showed that miR-124 expression was suppressed in patients with epilepsy and rats after drug induced-seizures. Intrahippocampal administration of a miR-124 duplex led to alleviated seizure severity and prolonged onset latency in two rat models (pentylenetetrazole- and pilocarpine-induced seizures), while miR-124 inhibitor led to shortened onset latency in pilocarpine-induced seizure rat models. Moreover, the result of local field potentials (LFPs) records further demonstrated miR-124 may have antiepilepsy function. Inhibition of neuronal firing by miR-124 was associated with the suppression of mEPSC, AMPAR- and NMDAR-mediated currents, which were accompanied by decreased surface expression of NMDAR. In addition, miR-124 injection resulted in decreased activity and expression of cAMP-response element-binding protein1 (CREB1). a key regulator in epileptogenesis. A dual-luciferase reporter assay was used to confirm that miR-124 targeted directly the 3'UTR of CREB1 gene and repressed the CREB1 expression in HEK293T cells. Immunoprecipitation studies confirmed that the CREB1 antibody effectively precipitated CREB1 and NMDAR1 but not GLUR1 from rat brain hippocampus. These results revealed a previously unknown function of miR-124 in neuronal excitability and provided a new insight into molecular mechanisms underlying epilepsy.

\section{Introduction}

Epilepsy is a collection of brain disorders that are characterised by recurrent unprovoked seizures and affect $1-2 \%$ of the world's population (Ref. 1). Regardless of its underlying causes, a seizure reflects an imbalance between inhibition and excitation that leads to abnormal hypersynchronous electrical activity of neuronal networks (Ref. 2). Recent study has revealed that epilepsy is associated with a cascade of molecular, cellular, and structural alterations (Ref. 3). However, most currently used anti-epileptic drugs (AEDs) fail to prevent or cure the disease, and $20-30 \%$ of patients remain refractory to treatment (Refs 4,5$)$.

MicroRNAs (miRNAs) are endogenous 23-nucleotide RNAs that play an important role in gene regulation. The targets of miRNAs include mRNAs that encode transcription factors, components of the miRNA machinery, and other proteins involved in translational regulation (Refs 6, 7, 8). In vertebrates, distinct miRNAs are expressed in the brain than in any other tissue and play critical roles in multiple neurological diseases including epilepsy (Refs 9, 10, 11). In the nervous system, miRNAs are involved in the control of synaptic function and plasticity (Refs 12, 13, 14).
In pilocarpine-induced seizures, a robust, rapid, and transient increase in the levels of the primary transcript of miR-132 (pri-miR-132) is followed by a subsequent increase in the levels of mature miR-132 (Ref. 15). In vivo microinjection of miR-132 antagomirs results in a reduction of seizure-induced neuronal death (Ref. 16). Evidence showed that knockdown or over expression of miRNA may potentially improve functional outcomes in patients with neurological diseases (Refs 10, 17, 18).

MiR-124 is highly abundant in the brain, retina and spinal cord. The expression of miR-124 was first detected in differentiating neurons, suggesting a role for this molecule in neural development (Refs 19, 20, 21 ). A recent study showed that miR-124 could function at the growth cone or at synapses by modulating synaptic activity and neuronal connectivity (Ref. 22). More importantly, miR-124 is also involved in neurological diseases. For instance, miR-124 is downregulated in brain tumours (Refs 23, 24). In experimental autoimmune encephalomyelitis (EAE), which is an animal model of multiple sclerosis, miR124 is suppressed in activated microglia (Ref. 25). A potential role of miR-124 in Alzheimer's disease and 
TABLE 1.

CLINICAL CHARACTERISTIC OF TLE PATIENTS

\begin{tabular}{lllcll}
\hline Number & Age $(\mathrm{Y})$ & Sex $(\mathrm{M} / \mathrm{F})$ & Course $(\mathrm{Y})$ & AEDs before surgery & Resection tissue \\
\hline 1 & 23 & $\mathrm{M}$ & 4 & VPA, TPM, PHT & TNl \\
2 & 32 & $\mathrm{M}$ & 6 & VPA, CBZ, OXC & TNr \\
3 & 24 & $\mathrm{M}$ & 17 & PB, PHT, TPM & TNr \\
4 & 31 & $\mathrm{~F}$ & 12 & CBZ, VPA, TPM & TNr \\
5 & 45 & $\mathrm{M}$ & 10 & TPM, LTG, CBZ & TN1 \\
6 & 30 & $\mathrm{M}$ & 6 & VPA, TPM, GBP & TNl \\
7 & 17 & $\mathrm{~F}$ & 11 & CBZ, TPM, CBZ, LTG, PHT & TNr \\
8 & 38 & $\mathrm{M}$ & 12 & VPA, TPM, OXC & TNl \\
9 & 29 & $\mathrm{~F}$ & 14 & VPA, PB, LTG, PHT & TNr \\
10 & 47 & $\mathrm{~F}$ & $\mathrm{TPM}$, LTG, CBZ, OXC & TN1 \\
11 & 36 & $\mathrm{M}$ & 7 & VPA, CBZ, LTG & TN1 \\
12 & 22 & & & & \\
\hline
\end{tabular}

AEDs, antiepileptic drugs (taken before operation); CBZ, carbamazepine; F, female; GBP, gabapentin; LTG, lamotrigine; 1, left; M, male; OXC, oxcarbazepine; PB, phenobarbital; PHT, phenytoin; r, right; TN, temporal neocortex; TPM, topamax; VPA, valproic acid; y, year.

Parkinson's disease has been recently suggested (Ref. 26). These results suggest that miR-124 may serve as a therapeutic target in neurological disorders. However, it remains unclear whether miR-124 is involved in regulation of neuronal excitability and seizures.

In the current study, we have observed that miR-124 is downregulated in epilepsy, as intrahippocampal supplementation with miR-124 inhibited neuronal firing and excitability, as well as susceptibility to epileptic seizures. Furthermore, we have identified that miR124 regulated the CREB1 gene and CREB1 protein expression. Thus, our findings provide valuable information towards unveiling the mechanisms of human temporal lobe epilepsy (TLE), and a novel target of potentially effective clinical therapies in the future.

\section{Materials and methods}

\section{Human subjects}

The patients with medically intractable TLE included in our study had typical clinical manifestations and characteristic electroencephalograms. Presurgical assessment consisted of obtaining a detailed history and neurological examination, interictal and ictal electroencephalogram studies, neuropsychological testing, and neuroradiological studies, such as magnetic resonance imaging (MRI).

Temporal neocortex tissues from 12 patients [7 males and 5 females; mean age, 31.17 \pm 9.15 years (range, 17-47 years); mean disease course, $11.08 \pm 4.64$ years (range, $4-18$ years)] were chosen at random among 223 specimens from our epilepsy brain tissue bank. All patients were refractory to the maximal dose of at least three AEDs (Table 1). We used temporal neocortex from patients treated for increased intracranial pressure because of head trauma tissue as control. The control subjects had no history of epilepsy or exposure to AEDs, and had no other neurological diseases [three males and three females; mean age, $28.33 \pm 13.98$ years (range,
15-54 years)] (Table 2). There were no significant differences in age and sex between the TLE and control groups $(P>0.05)$.

Each patient included in this study provided a signed consent form, and our study protocol complied with the guidelines for the performance of research involving human subjects, as established by the National Institutes of Health and the Committee on Human Research at the Chongqing Medical University. The research was conducted in accordance with the Declaration of Helsinki of the World Medical Association.

\section{Animals and treatment}

Administration of pilocarpine and intrahippocampal injection. All animal experiments complied with the guide for the Care and Use of Laboratory Animals of the Chongqing Medical University. Lithium-pilocarpine-induced seizures were as described previously (Refs 27, 28). Healthy adult male Sprague-Dawley rats (from the Chongqing Medical University Laboratory Animal Center) weighing 200-300 g were used as experimental subjects.

All rats were housed for 1 week before experiments were performed. The experimental group was divided randomly into the normal control group $(n=8)$ and the experimental groups $(n=40)$. The experimental groups were divided randomly into five subgroups

TABLE 2.

CLINICAL CHARACTERISTICS OF CONTROL GROUP

\begin{tabular}{llll}
\hline Number & Age $(\mathrm{Y})$ & Sex $(\mathrm{M} / \mathrm{F})$ & Resection tissue \\
\hline 1 & 24 & $\mathrm{M}$ & $\mathrm{TNl}$ \\
2 & 18 & $\mathrm{~F}$ & $\mathrm{TNr}$ \\
3 & 15 & $\mathrm{M}$ & $\mathrm{TNr}$ \\
4 & 27 & $\mathrm{~F}$ & $\mathrm{TNr}$ \\
5 & 32 & $\mathrm{~F}$ & $\mathrm{TNl}$ \\
6 & 54 & $\mathrm{M}$ & $\mathrm{TNl}$ \\
\hline
\end{tabular}

F, female; 1, left; M, male; r, right; TN, temporal neocortex; $y$, year. 
TABLE 3.

PRIMERS SEQUENCES USED FOR REAL-TIME PCR

\begin{tabular}{lll}
\hline Gene & Forward and reverse primers & Amplified fragment (bp) \\
\hline GAPDH & 5' CATCAAGAAGGTGGTGAAGCA 3' & 117 \\
CREB1 & 5' TCAAAGGTGGAGGAGTGGGT 3' & 104 \\
& 5' TGCAGACATTAACCATGACCA 3' & 104 \\
\hline
\end{tabular}

according to the period after onset of seizures: $6 \mathrm{~h}, 1$ day, 2 days, 3 days and 7 days. Rats in the experimental group were injected intraperitoneally with lithium chloride $(127 \mathrm{mg} / \mathrm{kg}$, i.p.; Sigma-Aldrich, St. Louis, MO, USA) $18 \mathrm{~h}$ before the first pilocarpine administration $(50 \mathrm{mg} / \mathrm{kg}$, i.p., Sigma-Aldrich, St. Louis, MO, USA). Pilocarpine $(10 \mathrm{mg} / \mathrm{kg}$, i.p.) was given repeatedly every $30 \mathrm{~min}$ until the rats developed seizures. Roughly 60 min after the onset of the status epilepticus, the animals were injected with diazepam $(10 \mathrm{mg} / \mathrm{kg}$, i.p.) to terminate seizures. Seizure activity was rated according to Racine's scale, and only those reached stages 4 or 5 were considered as being successfully kindled (Ref. 29). Control animals were given an intraperitoneal injection of saline.

Intrahippocampal injection was performed as described previously (Refs 30, 31, 32). The CY3tagged miR-124 mimics (agomir) and FAM-tagged miR-124 inhibitor (antagomir) and the scrambled control miRNA were provided by Guangzhou RiboBio Co., Ltd. (Guangzhou, China) (Ref. 33). miR-124 mimics and inhibitor were RNA duplex and were chemically modified and cholesterol conjugated from a hydoxyprolinol-linked cholesterol solid support and 2'-OMe phosphoramidites. Rats were given one treatment of miR-124 mimics ( $1 \mathrm{nM}$ in a total volume of $5 \mu \mathrm{l}$ ) (RiboBio, Guangzhou, China) or inhibitor ( $4 \mathrm{~nm}$ in a total volume of $5 \mu \mathrm{l}$ ) (RiboBio, Guangzhou, China) into the dorsal hippocampus (injection site: anterior/posterior, $-3.3 \mathrm{~mm}$; medial/lateral, $\pm 1.8 \mathrm{~mm}$; dorsal/ventral, $-2.6 \mathrm{~mm})$. Mimics-negative control sequences and inhibitor-negative control sequences were injected into the hippocampus as negative control. $72 \mathrm{~h}$ after intrahippocampal injection, the rats pre-treated by miR-124 mimics, inhibitor and scrambled controls were treated by pilocarpine administration. One day post pilocarpine injection, hippocampal tissues were obtained from rats.

To detect the expression of the miR-124 mimics and inhibitor, tissues were sectioned and mounted directly on slides. The intensity of fluorescence was directly detected using a laser scanning confocal microscope (Leica Microsystems Heidelberg GmbH, Germany) equipped with a Fluoview FVX confocal scanning head.

Administration of pentylenetetrazole (PTZ). Healthy adult male Sprague-Dawley rats (from the Chongqing Medical University Laboratory Animal Center) weighing 200-300 g were used as experimental subjects. The experimental group was divided randomly into the normal control group $(n=8)$ and the experimental groups $(n=16)$. The experimental groups were divided randomly into the mimics injection group and the mimics control injection group. Seizures were induced by i.p. injection of PTZ $(50 \mathrm{mg} / \mathrm{kg})$. At a dose of PTZ of $50 \mathrm{mg} / \mathrm{kg}$, rats developed seizures and had recovered fully by the time of sacrifice (Ref. 34).

Western blot analysis. Western blot analysis was performed as described previously (Ref. 35). Total and membrane proteins were extracted according to the manufacturers' instructions (Keygen Biotech, Nanjing, China for total proteins; Beyotime Institute of Biotechnology, Shanghai, China for membrane proteins). Protein concentrations were determined using the Enhanced BCA Protein Assay Kit (Beyotime Institute of Biotechnology, Shanghai, China). A total of $50 \mu \mathrm{g}$ of protein was loaded and then separated by SDS-PAGE (5\% spacer gel; $10 \%$ separating gel). The primary antibodies used were as follows: rabbit anti-pCREB (1:500, Cell Signaling, Boston, USA),

TABLE 4.

PRIMERS SEQUENCES USED FOR FUSION-PCR

\begin{tabular}{ll}
\hline Gene & Forward and reverse primers \\
\hline CREB-F & $5^{\prime}$ GCGCTCGAGGTTCCAACACCTGCCTCCA $3^{\prime}$ \\
CREB-R & $5^{\prime}$ AATGCGGCCGCTGGTGGTGGTATGTAAGTG $3^{\prime}$ \\
CREB-mut-F & $5^{\prime}$ TCCTTGATACGGAATGGGACAGAATACCCCAG 3' \\
CREB-mut-R & $5^{\prime}$ CTGTCCCATTCCGTATCAAGGAGCTCAGCACAA 3' \\
NR1-F & $5^{\prime}$ CGGGCGATCGCGTCAGCACCGTGGTGTGAG 3' \\
NR1-R & $5^{\prime}$ AATGCGGCCGCCACAGACCGAAAACTGCGT $3^{\prime}$ \\
NR1-mut-F & $5^{\prime}$ CCGAGCGCCACGGAACCCCGTGCGGCCCGTGCG 3' \\
NR1-mut-R & $5^{\prime}$ GCACGGGGTTCCGTGGCGCTCGGGCCCTGGGAG 3' \\
\hline
\end{tabular}



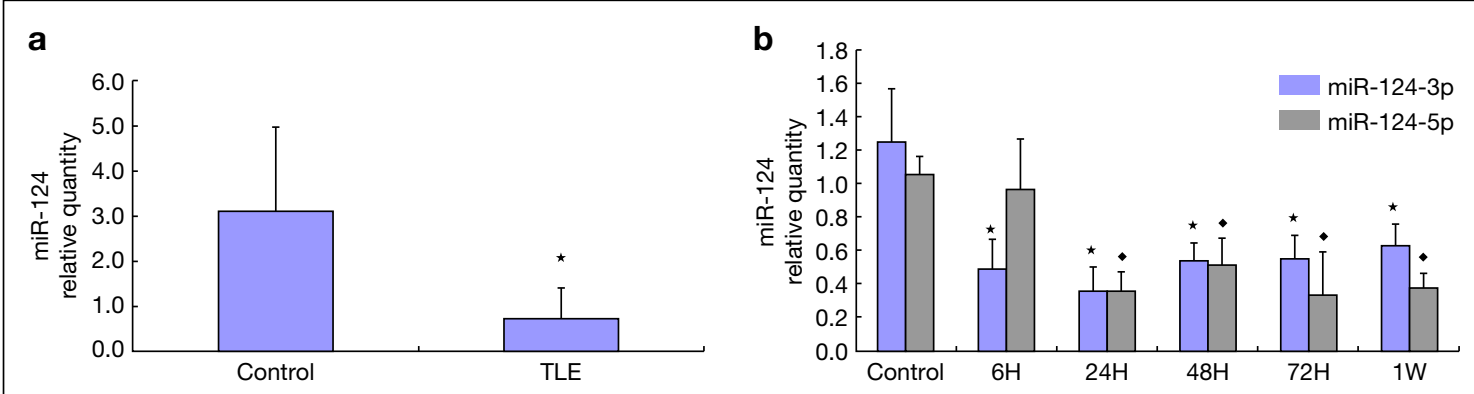

\section{qRT-PCR analysis of miR-124 expression in the hippocampus of patients with TLE and rat models}

Expert Reviews in Molecular Medicine @ 2016 Cambridge University Press

FIGURE 1

qRT-PCR analysis of miR-124 expression in the hippocampus of patients with TLE and rat models. (a) Relative quantity of mir-124 in the temporal neocortex of controls and patients with TLE. (b) Relative quantity of miR-124-3P and miR-124-5P in the hippocampus of rat models in control conditions and at different time points after seizure. ${ }^{*} P<0.05$, compared with the control.

rabbit anti-NMDAR1 (1:500, Epitomics, Burlingame, USA), rabbit anti-GLUR1 (1:500, Epitomics, Burlingame, USA) and rabbit anti- $\beta$-actin (1:4000, Beijing 4A Biotech Co., Ltd, Beijing, China). After 4 washes with Tween-20/Tris-buffered saline, the membranes were incubated with a horseradish peroxidase (HRP)-conjugated secondary antibody (1:4000, rabbit anti-goat IgG-HRP, Zhongshan Golden Bridge, Inc, Beijing, China). The resulting protein bands were visualised using an enhanced chemiluminescence substrate kit (Beyotime Institute of Biotechnology, Nantong, China) before digital scanning (Bio-Rad Laboratories, California, USA). The resultant pixel density was quantified using the Quantity One software (Bio-Rad Laboratories, California, USA) (Ref. 36).

Immunoprecipitation. Immunoprecipitation was used to survey the binding status between CREB1 and NMDAR1 or GLUR1. Approximately $100 \mathrm{mg}$ of hippocampaus tissues was homogenised and added RIPA lysis buffer (Beyotime, Nantong, China). Equal amounts of the proteins were incubated with $2 \mu \mathrm{l}$ of Rabbit IgG (1:100, Abcam, Cambridge, UK) as polyclonal-Isotype control or $10 \mu \mathrm{l}$ of CREB1 (1:20, Santa Cruz, Dallas, USA) or $4 \mu$ l of GLUR1 (1:50, Abcam, Cambridge, UK) or $4 \mu \mathrm{l}$ of NMDAR1 (1:50, Cell Signal, Boston, USA) antibody $8 \mathrm{~h}$ at $4^{\circ} \mathrm{C}$ followed by incubation of $20 \mu \mathrm{l}$ of Protein A + G Agarose beads (Beyotime, Nantong, China) overnight at $4^{\circ} \mathrm{C}$. The mixture was centrifuged $\left(3000 \mathrm{rpm}, 5 \mathrm{~min}, 4^{\circ} \mathrm{C}\right)$ and rinsed hree times with RIPA lysis buffer. The mixture was boiled with $1 \times$ Western blot loading buffer for $5 \mathrm{~min}$. After spinning $\left(3000 \mathrm{rpm}, 5 \mathrm{~min}, 4^{\circ} \mathrm{C}\right)$, the supernatants were subjected to Western blot with same set of antibodies as above (Ref. 37).

Quantitative PCR analysis. Total RNA was extracted using the PrimeScript ${ }^{\mathrm{B}}$ RT reagent Kit according to the manufacturer's instructions (TaKaRa, Dalian, China). Real-time PCR was performed using a SYBR $^{\circledR}$ Premix Ex Taq ${ }^{\mathrm{TM}}$ Kit (TaKaRa, Dalian, China) and the iQ5 Real-Time PCR detection system (Bio-Rad Laboratories, California, USA). The PCR primers used for the amplification of the CREB1 mRNA are listed in Table 3. qRT-PCR was used to analyse the expression of miR-124-3p (miR-124-3p: UAAGGCACG CGGUGAAUGCC) and miR-124-5p (miR-124-5p: CGUGUUCACAGCGGACCUUGAU) and U6 snRNA using the Bulge-LoopTM miRNA qPCR Primer Set (RiboBio, Guangzhou, China) according to the manufacturer's instructions. The expression level of CREB1 relative to that of GAPDH and the expression level of miR-124 relative to that of U6 were determined (Relative Quantity $=2^{-\Delta \Delta \mathrm{CT}}$ ) (Ref. 38).

Immunohistochemistry. pCREB location and expression were detected by site-specific immunohistochemical staining (Ref. 39). The primary antibody used was a rabbit polyclonal anti-pCREB antibody (1:800, Cell Signal, Boston, USA). Sections were then incubated with a biotinylated goat anti-rabbit secondary antibody (streptavidin-peroxidase kits; Zhongshan Golden Bridge, Inc, Beijing, China). Finally, sections were treated with $\mathrm{ABC}$ solution at $37^{\circ} \mathrm{C}$ for $30 \mathrm{~min}$, washed with PBS, and incubated with DAB (3,3'-diaminobenzidine; Zhongshan Golden Bridge, Inc, Beijing, China) for $3 \mathrm{~min}$. As a negative control, the primary and secondary antibodies were replaced with PBS. An OLYMPUS PM20 automatic microscope (Olympus, Osaka, Japan) was used to collect the images.

Electrophysiology. The rats pre-treated by miR-124 mimics and mimics control were collected $72 \mathrm{~h}$ after intrahippocampal injection then followed by pilocarpine administration. One day post pilocarpine injection, hippocampal slices were obtained from rats. Coronal 
brain slices $(350 \mu \mathrm{m})$ were obtained in ice-cold sterile slice solution (containing, in $\mathrm{mm}$ : $\mathrm{KCl}, 2.5$; $\mathrm{NaH}_{2} \mathrm{PO}_{4} \cdot 2 \mathrm{H}_{2} \mathrm{O}, 1.25 ; \mathrm{MgCl}_{2} \cdot \mathrm{H}_{2} \mathrm{O}, 6 ; \mathrm{CaCl}_{2}, 1$; $\mathrm{NaHCO}_{3}, 26$; sucrose, 220; and glucose, 10). Slices were perfused with artificial cerebral spinal fluid (ACSF) at $35^{\circ} \mathrm{C}$ for $1 \mathrm{~h}$, and then at room temperature. The ACSF was saturated with a mixture of $95 \% \mathrm{O}_{2}$ and $5 \% \mathrm{CO}_{2}$ at $\mathrm{pH} 7.4$ (Ref. 40).

To measure cell excitability, the whole-cell currentclamp technique was used to record action potentials in CA1 region (5 neurons each group) (Refs 41, 42). The internal solution contained (in mM): $60 \mathrm{~K}_{2} \mathrm{SO}_{4}, 60$ NMG, 40 HEPES, $4 \mathrm{MgCl}_{2}$, 0.5 BAPTA, 12 phosphocreatine, $2 \mathrm{Na}_{2} \mathrm{ATP}$ and $0.2 \mathrm{Na}_{3} \mathrm{GTP}$ ( $\mathrm{pH}$ 7.2-7.3; 265-270 mOsm). To simulate human epilepsy, a magnesium-free solution was applied in ACSF for $1 \mathrm{~h}$. During this time, the neuronal cells underwent sustained seizure activity (Ref. 43). The slice was then bathed with normal ACSF for the remainder of the recordings.

For mEPSC recording, the internal solution was composed of (in mM): 130 Cs-methanesulfonate, 10 HEPES, $10 \mathrm{CsCl}, 4 \mathrm{NaCl}, 1 \mathrm{MgCl}_{2}, 1$ EGTA, 5 NMG, $5 \mathrm{MgATP}, 0.5 \mathrm{Na}_{2} \mathrm{GTP}$ and 12 phosphocreatine (pH 7.2; 275-290 mOsm). Tetrodotoxin (TTX, $1 \mu \mathrm{M}$ ) and Bicuculline $(10 \mu \mathrm{M})$ were added to block GABA activity. To evaluate NMDAR/AMPAR-EPSC in hippocampal neurons, the whole-cell voltage-clamp recording technique was used (Ref. 41). A patch electrode $(3-5 \mathrm{M} \Omega)$ was filled with an internal solution containing (in $\mathrm{mm}$ ): 130 Cs-methanesulfonate, 10 HEPES, $10 \mathrm{CsCl}, 4 \mathrm{NaCl}, 1 \mathrm{MgCl}_{2}, 1$ EGTA, $5 \mathrm{~N}$ methyl-D-glucamine, 12 phosphocreatine, 5 MgATP and $0.5 \mathrm{Na}_{2} \mathrm{GTP}$ ( $\mathrm{pH} 7.2 ; 275-290 \mathrm{mOsm}$ ). Slices were bathed in ACSF containing bicuculline $(10 \mu \mathrm{M})$ to block GABAA receptors. Evoked currents were generated using a $0.1 \mathrm{~Hz}$ pulse (intensity, 50-200 $\mu \mathrm{A}$; duration, $400 \mu \mathrm{s})$ delivered by a stimulation isolation unit that was controlled by an S48 pulse generator (AstroMed). A bipolar stimulating electrode (FHC) was positioned $\sim 150 \mu \mathrm{m}$ from the neuron being recorded. The membrane potential of recorded neurons was first held at $+40 \mathrm{mV}$, and the presynaptic simulation elicited dual component responses (mediated by both AMPARs and NMDARs). After obtaining 30 traces as the baseline, the NMDAR-selective antagonist D-APV $(50 \mu \mathrm{M})$ was applied; thus, pure AMPARmediated EPSCs were obtained. The subtraction of the AMPAR EPSCs from the dual component EPSCs generated the NMDAR EPSCs.

A Multiclamp 700B amplifier (Axon, Sunnyvale, CA, USA) and Digidata 1322A were used to collect and analyse data, which were filtered at $10 \mathrm{kHz}$ and low-pass filtered at $2 \mathrm{kHz}$, followed by recording using the pClamp 9.2 software (Molecular Devices, Sunnyvale, CA, USA). The Mini Analysis program (Synaptosoft, Leonia, NJ) was used to analyse synaptic activity. Data were collected after currents had been stable for 5-15 min. Results were discarded if the series resistance changed by $>15 \%$.
In vivo multichannel electrophysiological recording. Local field potentials (LFPs) analysis was performed as described previously (Refs 44, 45). After anesthetised by chloral hydrate $(350 \mathrm{mg} / \mathrm{kg}$, i.p.), the rats were received one treatment of miR-124 mimics or inhibitor or scrambled controls by intrahippocampal injection (anterior/posterior, $-3.3 \mathrm{~mm}$; medial/ lateral, $\pm 1.8 \mathrm{~mm}$; dorsal/ventral, $-2.6 \mathrm{~mm})$. Then a recording micro wire array $(4 \times 4$ array of platinumiridium alloy wire, each with $25 \mu \mathrm{m}$ diameter, Plexon, Dallas, TX) was implanted into the right dorsal hippocampus (anterior/posterior, $-3.7 \mathrm{mM}$; medial/lateral, $-2.5 \mathrm{~mm}$; dorsal/ventral, $-2.7 \mathrm{~mm}$ ). The rats which were treated surgery were recovered for 5 days before the electrophysiological activity was recorded. OmniPlex ${ }^{\circledR} \mathrm{D}$ neural Data Acquisition System (Plexon, Dallas, TX) was used recording for LFPs. The LFPs signals were digitised at $4 \mathrm{kHz}$, filtered $(0.1-1000 \mathrm{~Hz})$ and preamplified $(1000 \times)$. Neuro Explorer v4.0 (Plexon, Dallas, TX) was used for the LFPs data analysis. Seizures were induced in rats by lithium chloride-pilocarpine and the electrophysiological was continuously recorded (more than $80 \mathrm{~min}$ ). A typical seizure discharged of electrophysiological was showing as high-frequency (frequency $>5 \mathrm{~Hz}$ ), high-amplitude discharge (amplitude $>2$ times the baseline) and lasting longer than $5 \mathrm{~s}$ (Ref. 44).

Luciferase reporter assay. To confirm the hypothesis that miR-124 targets the $3^{\prime} \mathrm{UTR}$ region of CREB1, a dual-luciferase reporter assay was used to determine whether miR-124 targeted directly the $3^{\prime}$ UTR of CREB1 gene and repressed the CREB1 expression in human-type cells.

A 804-bp segment from the $3^{\prime}$ UTR of the CREB1 gene containing the two miR-124 binding sites was produced by PCR with the forward primer $5^{\prime}$ GCGCTCGAGGTTCCAACACCTGCCTCCA- $3^{\prime}$ and the common reverse primer $5^{\prime}$-AATGCGGCCGCT GGTGGTGGTATGTAAGTG-3', and then cloned into the Xhol/NotI site of pmiR-RB-REPORT ${ }^{\mathrm{TM}}$ (RiboBio, Guangzhou, China). For mutant construct of CREB1 3'UTR, deletion mutagenesis and fusionPCR were performed. Four fragments, including two mutant miR-124 binding sites and two middle segments, were firstly produced by PCR; the primers are shown in Table 4. The full length of CREB1 promoter, containing the two mutant miR-124 binding sites, was obtained by mixing the two fragments produced from the first-step PCR and then using them as the template in the second PCR reaction with the outermost primers, and then cloned into the XhoI/NotI site of pmiR-RB-

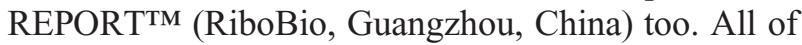
the constructs were confirmed by sequencing.

HEK293T cells were obtained from institute of Biochemistry and Cell Biology (Chinese Academy of Sciences, Shanghai, China.). Cells $\left(5 \times 10^{4}\right.$ cells per well) were plated in 24 -well plates $24 \mathrm{~h}$ before transfection and were maintained in DMEM/F12 (HyClone, 
a
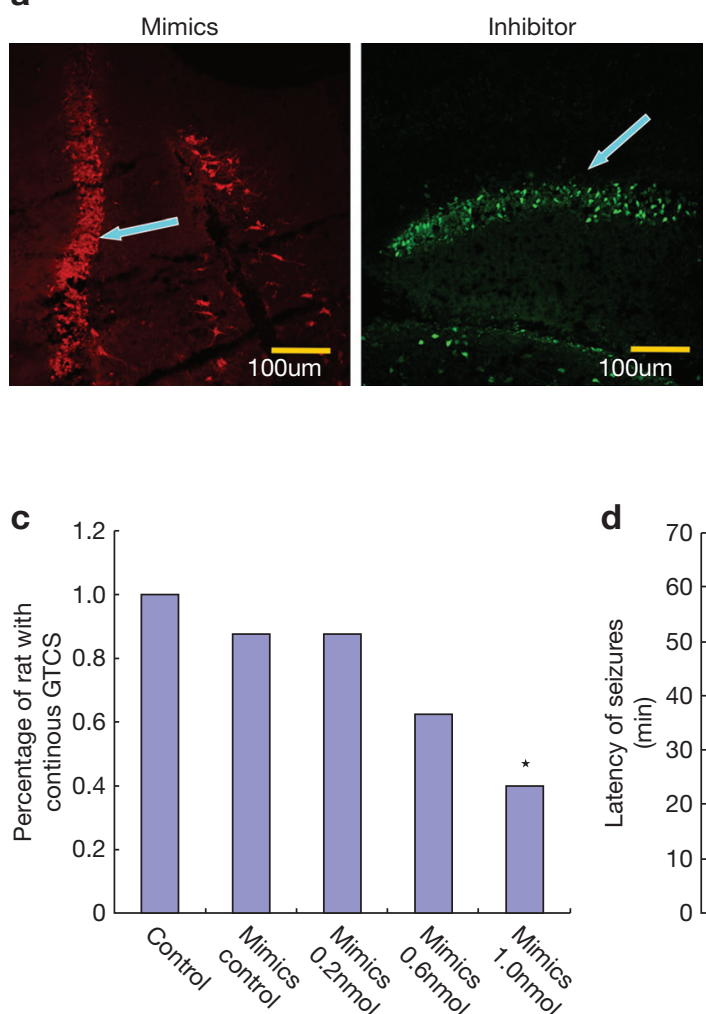

e
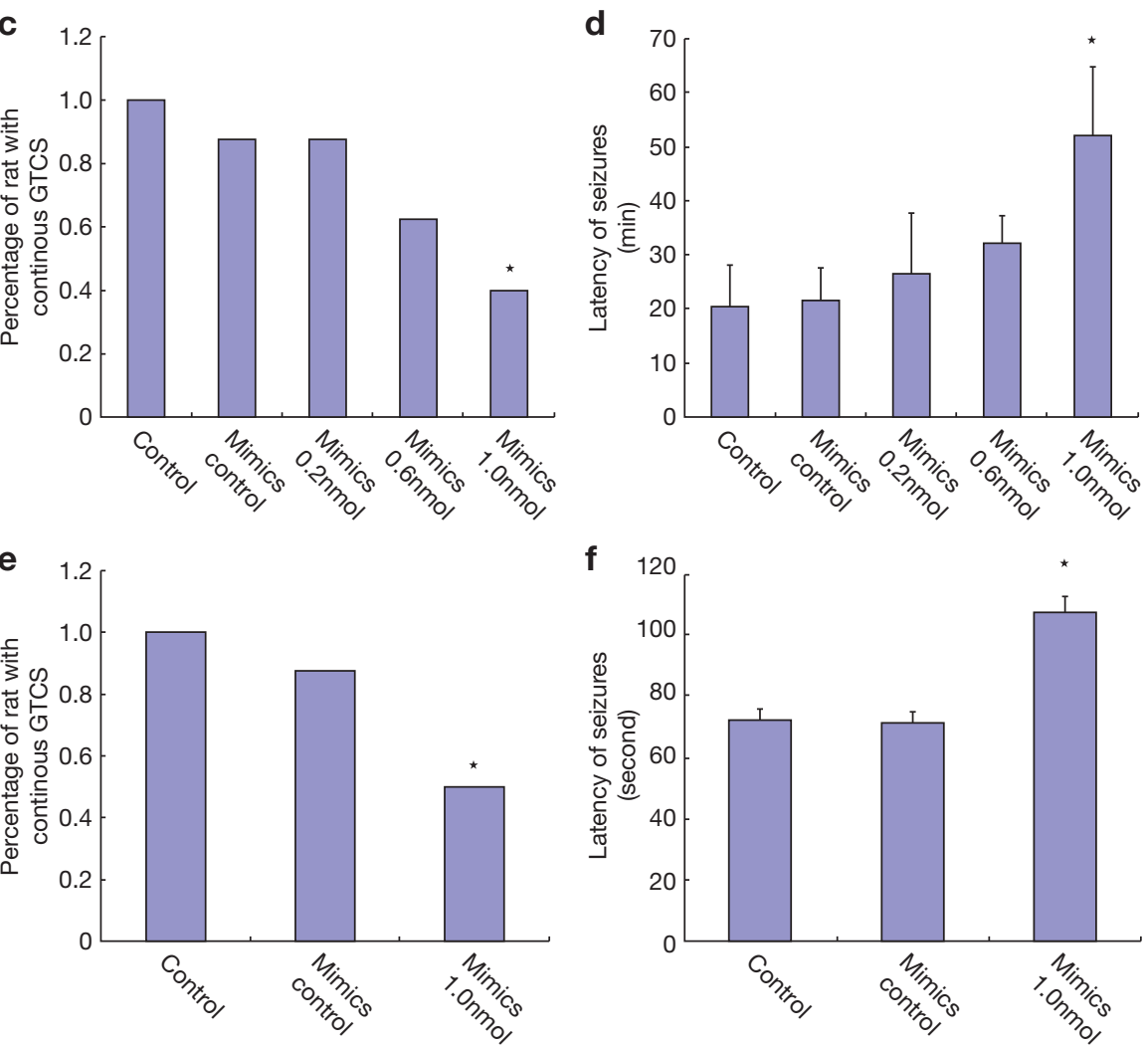

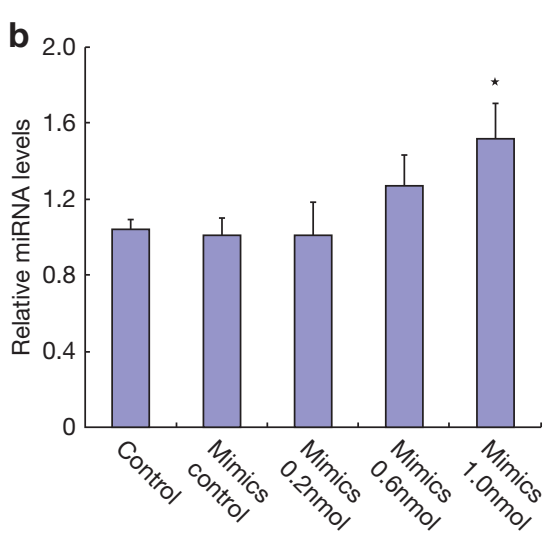


UT, USA) plus 10\% foetal bovine serum (Gibco, NY, USA). The CY3 labelled mimics/negative control were synthesised by RiboBio (RiboBio, Guangzhou, China). HEK293T cells were co-transfected with $100 \mathrm{ng} / \mathrm{ml} \quad$ pmiR-RB-REPORT $^{\mathrm{TM}} \quad$ (RiboBio, Guangzhou, China), including the $3^{\prime}$ UTR of CREB1 [with either wild-type (WT) or mutant-type miR-124 binding sites] and miR mimics or control (RiboBio, Guangzhou, China) at a final concentration of $100 \mathrm{~nm}$ using riboFECTTM CP as described by the manufacturer. Luciferase assays were performed with a DualLuciferase Reporter Assay System (Promega, Madison, USA) $48 \mathrm{~h}$ after transfection. Renilla luciferase activity was normalised to that of firefly luciferase (Ref. 46, $47,48)$.

Moreover, to investigate whether NMDAR1 was also a direct target of miR-124, a 946-bp segment from the $3^{\prime}$ UTR of the NMDAR1 gene containing the two miR-124 binding sites was produced by PCR and then a Dual-Luciferase Reporter Assay System (Promega, Madison, USA) was performed as described previously.

Statistical analyses. Data were expressed as means \pm standard deviation (SD). All statistical analyses were conducted using the statistical software SPSS 13.0. Data involving more than two groups were assessed by one-way ANOVA, followed by Tukey's HSD post hoc multiple comparison test. Student's $t$-test was used for statistical analysis of differences between the TLE group and the control group in humans. $P<$ 0.05 was considered statistically significant.

\section{Results}

miR-124 expression is decreased in the temporal neocortex of patients with epilepsy and in a rat model

First we investigated the expression of miR-124 in the temporal neocortex in patients with TLE by qRT-PCR. The results showed the expression of miR-124 was significantly lower in patients with TLE than it was in controls (Fig. 1a). To test whether miR-124 is also downregulated in the hippocampus of rats after pilocarpine-induced seizures, we next determined miR-124 expression in the hippocampus of this rat model; the results showed that the relative quantity of miR-124$3 p$ was significantly decreased at $6 \mathrm{~h}$ after pilocarpine-induced seizures and remained significant low for up to 1 week (Fig. 1b). Similarly, the relative quantity of miR-124-5p was also significantly decreased at $24 \mathrm{~h}$ after pilocarpine-induced seizures and remained significant low for up to 1 week compared with control (Fig. 1b).

\section{Effect of miR-124 mimics on seizure activity}

After the injection of miR-124 mimics (agomir) as opposed to scrambled miRNA mimics, the miR-124 mimics was observed in the dentate gyrus (DG) and CA3-CA1 region of the rat hippocampus (Fig. 2a). The optimal dose that led to significant expression of miR-124 (40-50\% enhancement) was $1.0 \mathrm{~nm}$ (Fig. 2b), which did not result in any behavioural abnormalities before seizures was induced.

To test whether miR-124 interferes with the seizure phenotype, we measured the effect of miR-124 mimics on seizure activity. After the injection of pilocarpine, behavioural seizures were detected in each group. Compared with the control group, $1.0 \mathrm{~nm}$ mimics led to a significant decrease in the incidence of generalised tonic-clonic seizures (GTCS, Fisher's exact test, $P=0.038$, Fig. 2c). In addition, $1.0 \mathrm{~nm}$ miR-124 mimics significantly delayed seizure onset, as measured by latency. The differences in latency between the $1.0 \mathrm{~nm}$ mimics, the control, and the mimics control groups were statistically significant (52.25 $\pm 12.66 \mathrm{~min}$ in $1.0 \mathrm{~nm}$ the mimics group, $n=8$; $20.43 \pm 7.62 \mathrm{~min}$ in the control group, $n=8$; and $21.63 \pm 6.02 \mathrm{~min}$ in the mimics control group, $n=8$; $P<0.05$; Fig. 2d).

We then studied whether preinjection of a miR-124 mimics affected PTZ-induced seizures in rat models. After injection of PTZ, behavioural seizures were detected in each group. Compared with the control group, $1.0 \mathrm{~nm}$ mimics led to a significant decrease in the incidence of generalised tonic-clonic seizures (Fisher's exact test, $P=0.021$, Fig. 2e). In addition, $1.0 \mathrm{~nm}$ miR-124 mimics delayed seizure onset significantly, as measured by latency. The differences in latency between the $1.0 \mathrm{~nm}$ mimics, the control, and

\section{FIGURE 2}

Effect of a miR-124 mimics and inhibitor on rat seizure behavioural activities. (a) Fluorescent image showing positive expression of the miR124 mimics and miR-124 inhibitor in the DG of the hippocampus. The blue arrow indicates a granule cell; Scale bar, $100 \mu \mathrm{m}$. (b) miR-124 levels in the hippocampus $72 \mathrm{~h}$ after intrahippocampal injection of the miR-124 mimics $(0.2 / 0.6 / 1.0 \mathrm{~nm})$ and mimics control $(1.0 \mathrm{nM}) .{ }^{*} P<0.05$, compared with the control, $n=3$ in each group. (c) Effect of intrahippocampal injection of the miR-124 mimics $(0.2 / 0.6 / 1.0 \mathrm{~nm})$ and mimics control $(1.0 \mathrm{nM})$ on the percentage of rats with generalised tonic-clonic seizures (GTCS) in pilocarpine-induced seizure rat models. ${ }^{*} P<0.05$, compared with the control, $n=8$ in each group. (d) Effect of intrahippocampal miR-124 mimics $(0.2 / 0.6 / 1.0 \mathrm{~nm})$ and mimics control $(1.0 \mathrm{nM})$ injection on the latency of seizures in pilocarpine-induced rat models. ${ }^{*} P<0.05$, compared with the control, $n=8$ in each group. (e) Effect of intrahippocampal miR-124 mimics (1.0 nM) and mimics control (1.0 nM). injection on the percentage of rats with generalised tonic-clonic seizures (GTCS) in PTZ-induced seizure rat models. ${ }^{*} P<0.05$, compared with the control, $n=8$ in each group. (f) Effect of intrahippocampal miR-124 mimics (1.0 nM) and mimics control (1.0 nM) injection on the latency of seizures in PTZ-induced seizure rat models. ${ }^{*} P<$ 0.05 , compared with the control, $n=8$ in each group. $(\mathrm{g})$ miR-124 levels in the hippocampus $72 \mathrm{~h}$ after intrahippocampal injection of the miR124 inhibitor (4.0 nM) and inhibitor control (4.0 nM). ${ }^{*} P<0.05$, compared with the control, $n=5$ in each group. (h) No statistically considerable differences was found between the percentage of rats with GTCS in control, inhibitor control and inhibitor groups. $n=8$ in each group. (i) Effect of intrahippocampal miR-124 inhibitor (4.0 nM) and inhibitor control (4.0 nM) injection on the latency of seizures in pilocarpine-induced rat models. ${ }^{*} P<0.05$, compared with the control, $n=8$ in each group. 


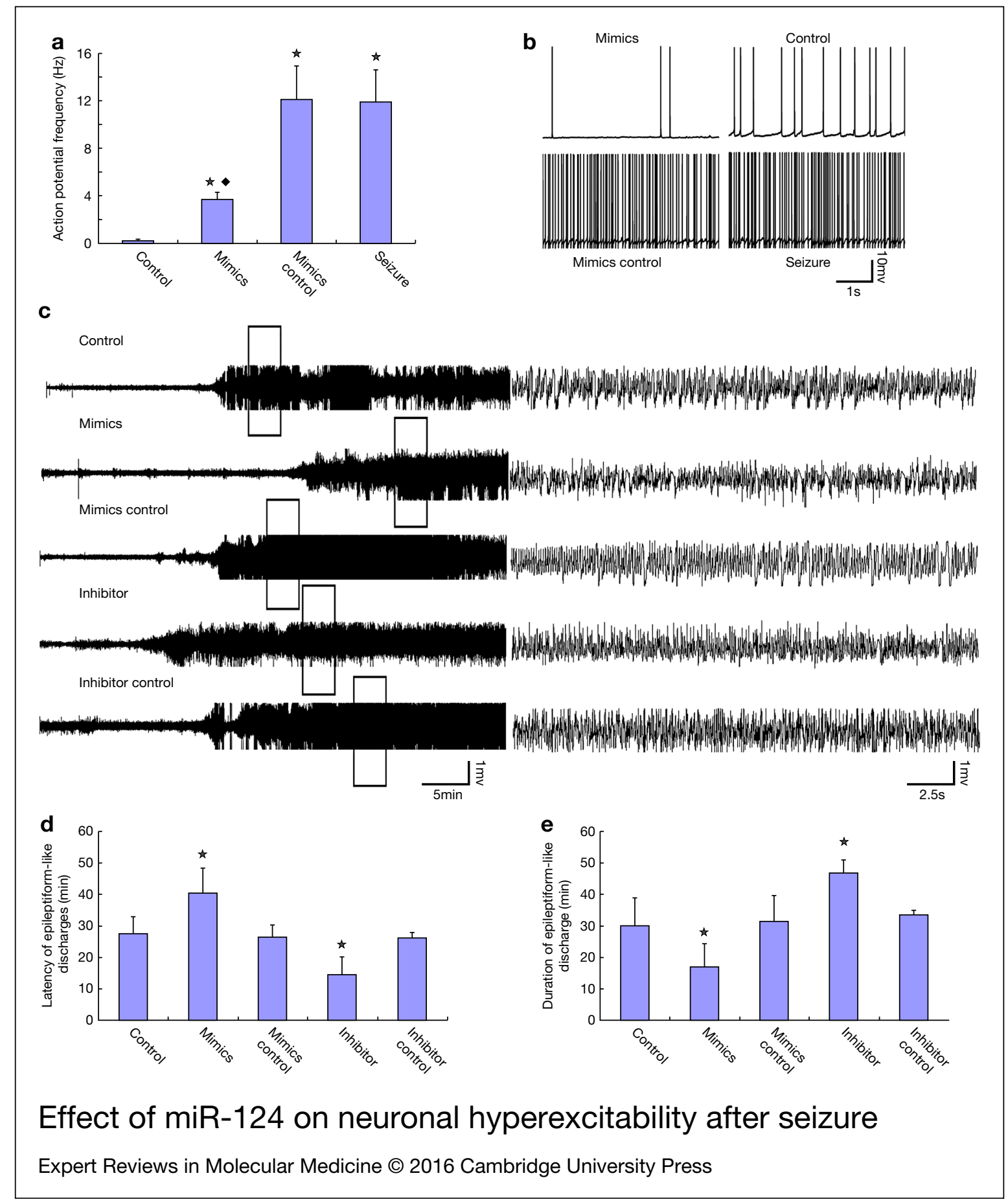

FIGURE 3.

Effect of miR-124 on neuronal hyperexcitability after seizure. (a) Changes of AP frequency. ${ }^{*} P<0.05$, compared with control, $n=5$ in each group; $P<0.05$, compared with the mimics control, $n=5$ in each group. (b) Representative traces of action potential (AP) discharges. (c) Typical trace of LFPs on rats treated with miR-124 mimics and inhibitor. (d) The miR-124 mimics significantly prolonged the latency of epileptiform-like discharges and the miR-124 inhibitor significantly shorted the latency of epileptiform-like discharges compared with the controls in a model of pilocarpine induced seizures, $n=5, \star P<0.05$, compared with the control. (e) The duration of epileptiform-like discharges last shorter on rats treated with miR-124 mimics and longer on rat treated with miR-124 inhibitor in a model of pilocarpine-induced seizures, $n=5$, $\star P<0.05$, compared with the control.

the miR-124 mimics control groups were statistically significant $(107.25 \pm 10.24 \mathrm{~s}$ in the $1.0 \mathrm{~nm}$ miR-124 mimics group, $n=8 ; 72.38 \pm 10.60 \mathrm{~s}$ in the control group, $n=8 ; 71.14 \pm 9.74 \mathrm{~s}$ in the mimics control group, $n=8 ; P<0.05$; Fig. 2f).
Effect of miR-124 inhibitor on seizure activity

Similar methods were used for transfection of miRNA inhibitor (antagomir) into hippocampus of rats. After the injection of miR-124 inhibitor as opposed to scrambled miRNA inhibitor, the miR-124 inhibitor 


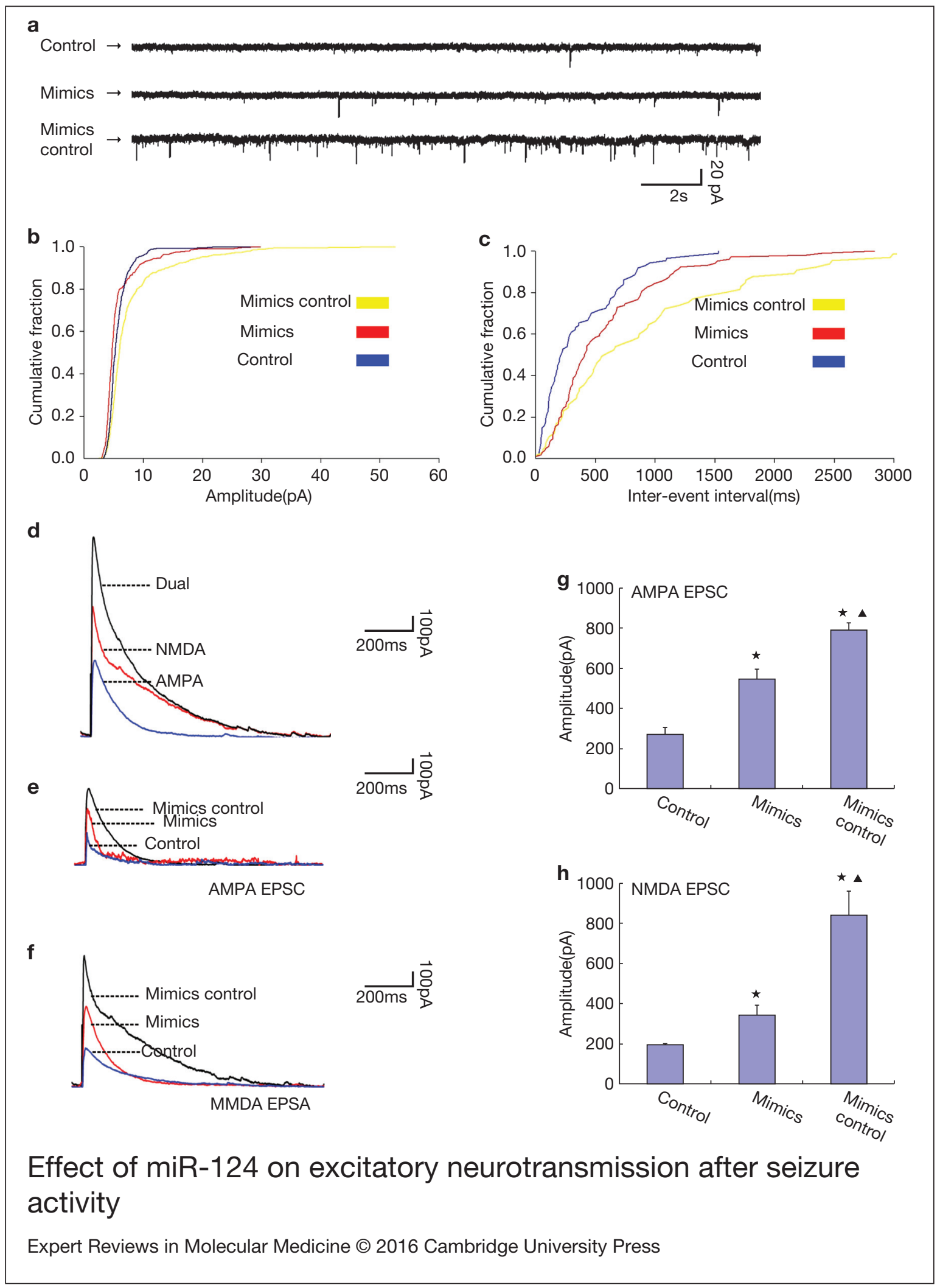

FIGURE 4

Effect of miR-124 on excitatory neurotransmission after seizure activity. (a) Representative traces of mEPSC recorded in hippocampal pyramidal cells in control (without seizure inducing) group, mimics group and mimics control group. (b, c) Cumulative fractions of amplitude and interevent interval ( $n=5$ in individual groups). (d) Representative traces showing dual components (mediated by AMPARs and NMDARs, as indicated) were recorded from CA1 neurons by holding the membrane potential at $+40 \mathrm{mV}$. (e, f) Sample traces showing AMPAR- (e) and NMDAR- (f) mediated components in control (without seizure inducing) and mimics and mimics control injection in pilocarpineinduced seizure rats, respectively. (g) Summary of AMPAR-mediated currents. (h) Summary of NMDAR-mediated currents. ${ }^{*} P<0.05$, compared with the control $(n=5)$. $\mathbf{\Delta} P<0.05$, compared with the mimics control, $n=5$. 


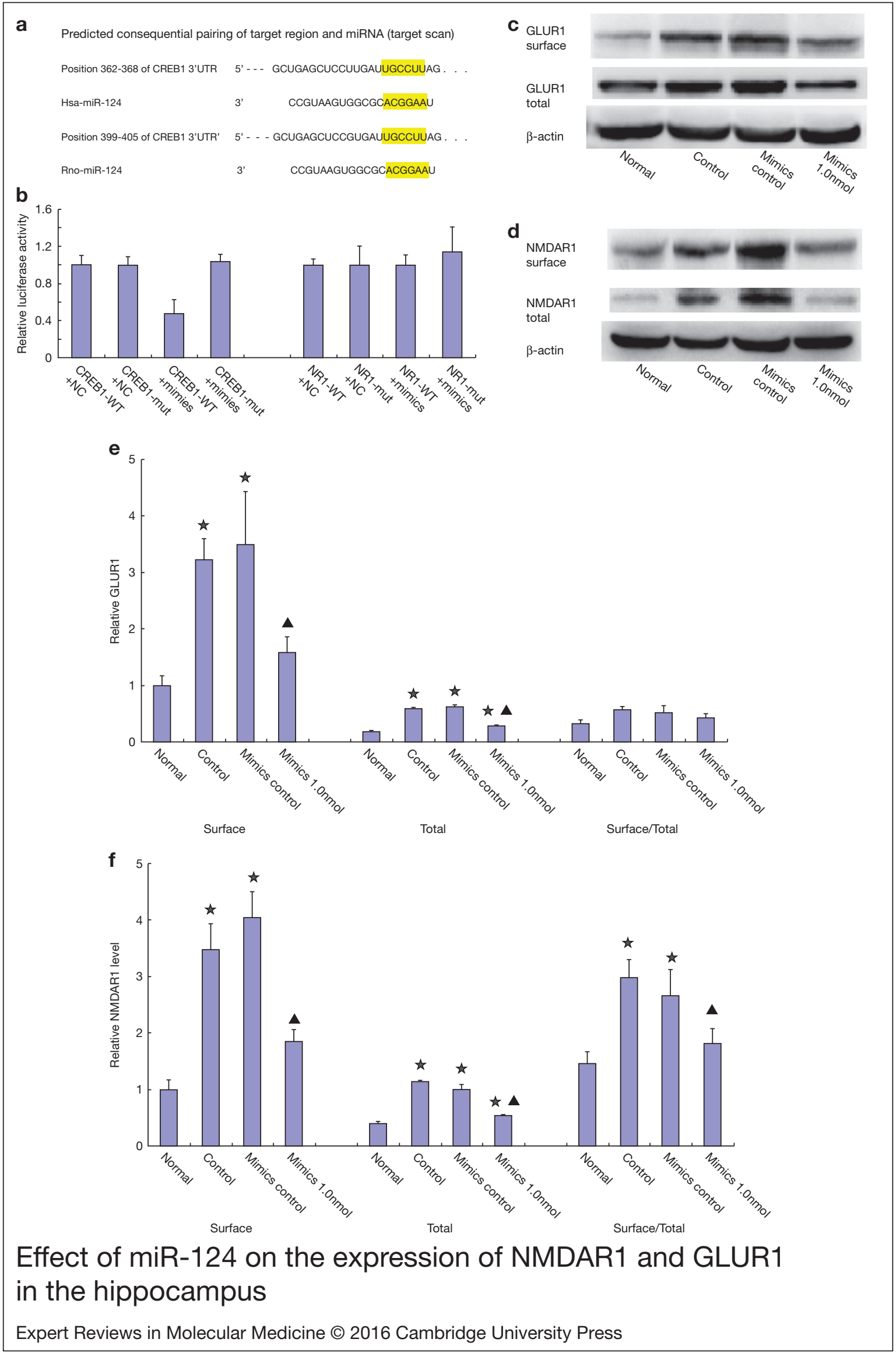


was observed in the DG and CA3-CA1 region of the rat hippocampus (Fig. 2a). $4.0 \mathrm{~nm}$ dose of miR-124 inhibitor was used which led to significant decrease of miR-124 level $(P<0.05$, Fig. $2 \mathrm{~g})$. To test whether miR-124 inhibitor interferes with the seizure phenotype, we also measured the effect of miR-124 inhibitor on seizure activity. After the injection of pilocarpine, behavioural seizures were detected in each group. No statistically considerable difference was observed in the incidence of generalised tonic-clonic seizures between the $4.0 \mathrm{~nm}$ inhibitor, the control and the inhibitor control groups $(P>0.05$, Fig. $2 \mathrm{~h})$. The differences in latency between the $4.0 \mathrm{~nm}$ inhibitor, the control and the inhibitor control groups were statistically significant (23.41 $\pm 6.24 \mathrm{~min}$ in the inhibitor control group, $n=8$; $20.98 \pm 4.35 \mathrm{~min}$ in the control group, $n=8$; and $15.98 \pm 4.05 \mathrm{~min}$ in the inhibitor group, $n=8 ; P<$ 0.05; Fig. 2i).

\section{miR-124 inhibits neuronal hyperexcitability after seizure activity}

To test whether the effect of miR-124 on behavioral activity was because of the inhibition of hyperexcitability, we measured action potentials in hippocampal CA1 neurons. As shown in Figure 3, slices from controls (without seizure inducing) exhibited relatively few action potentials. In the brains of rats with seizures induced by pilocarpine, slices with scrambled miRNA mimics control and seizure group showed an outburst of action potentials, whereas slices with $1.0 \mathrm{nM}$ miR-124 mimics exhibited significantly reduced action potential frequencies (Fig. 3a and b; mimics versus control, $P<0.05$; mimics versus scrambled mimics, $P<0.05$ ).

We further used an in vivo multichannel electrophysiological recording to record the effect of miR-124 on LFPs. The typical changes of LFPs in rat pre-treated by miR-124 mimics and inhibitor are shown in Figure 3c. We observed that treatment with miR-124 mimics dramatically prolonged the latency of epileptiform-like discharges (Fig. 3d, $P<0.05$ ) and shortened the duration of epileptiform-like discharges (Fig. 3e, $P<0.05$ ) in a rat model of pilocarpine-induced seizures. Moreover, rats injected with miR-124 inhibitor presented decreased latency of epileptiform-like discharges in a model of pilocarpine (Fig. 3d, $P<0.05$ ) while prolonged duration of epileptiform-like discharges (Fig. 3e, $P<0.05$ ). The result of LFPs on pilocarpine-induced seizures model further demonstrated miR-124 may have anti-epilepsy function.

To test if enhanced excitatory neurotransmission contributes to neuronal hyperactivity, mEPSC was recorded in the hippocampal slices. The effective dose of the miR-124 mimics significantly decreased the amplitude and frequency of mEPSC compared with the mimics control and control group $(P<0.05$; Fig. $4 a-c)$. To identify the AMPA receptor (AMPAR) or NMDA receptor (NMDAR) mediates inhibition of excitatory neurotransmission afforded by miR-124, we examined evoked APMAR and NMDAR currents in the brain slices. In the brain slices of rats with seizures induced by pilocarpine, both APMAR- and NMDAR-mediated currents were significantly increased compared with those observed in control group animals. However, both AMPARand NMDAR-mediated currents were significantly reduced after miR-124 mimics injection (Fig. 4d-h). These results suggest that miR-124 inhibits epileptic hyperactivities through APMAR- and NMDARmediated mechanisms.

\section{miR-124 Interacts directly with the $3^{\prime} U T R$ region of CREBI}

Using TargetScan (Release 4.2) online searching programs, we have identified CREB1 as the potential target of miR-124. A $100 \%$ matched sequence was found at the CREB1 mRNA 3'UTR (Fig. 5a).To confirm the hypothesis that miR-124 targets the $3^{\prime}$ UTR region of CREB1, the entire $3^{\prime} \mathrm{UTR}$ region of CREB1 was cloned into the XhoI/NotI site of pmiRRB-REPORT $^{\mathrm{TM}}$ and co-transfected the HEK293T cells with this vector along with either the miR-124 mimics or its negative control. Using the luciferase reporter system, we found that co-transfection of miR-124 mimics along with the $3^{\prime}$ UTR of WT CREB 1 caused a significant decrease by over $50 \%$ in luciferase units compared to controls (Fig. 5b). However, co-transfecion of miR-124 mimics not significantly suppressed the luciferase activity of reporter genes containing $3^{\prime}$ UTR of NMDAR1 compared with the control after statistics calculation (Fig. 5b). These results demonstrated that miR-124 targeted specifically the $3^{\prime}$ UTR region of CREB1.

\section{FIGURE 5 .}

Effect of miR-124 on the expression of NMDAR1 and GLUR1 in the hippocampus. (a) Diagram of the CREB1-3'-UTR with potential bindingsites for miR-124. (b) Relative luciferase activity of reporters, including WT or mutant CREB1 and NMDAR1 3'-UTR co-transfected with NC or miR-124 mimics. *indicates significant difference at $P<0.01$, respectively. (c) Sample Western blots showing surface and total levels of GLUR1 subunits in normal (without seizure inducing) and pilocarpine-induced seizure rats without treatment (control), and in rats treated with mimics control and mimics. (d) Sample Western blots showing the surface and total levels of expression of NMDAR1 subunits in normal (without seizure inducing) and pilocarpine-induced seizure rats without treatment (control), and in rats treated with mimics control and mimics. (e) Summary showing the surface expression and the total and surface/total ratios of GLUR1 subunits. ${ }^{*} P<0.05$, compared with the control. $\boldsymbol{\Delta} P<0.05$, compared with the mimics control, $n=3$. (f) Summary showing the surface expression and the total and surface/ total ratios of NMDAR1 subunits. $\mathbf{\Delta} P<0.05$, compared with the mimics control, $n=3$. 
a

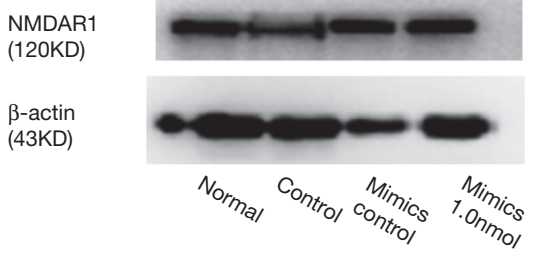

C

$\begin{array}{ll}\text { IP } & \text { (42KD) } \\ \text { IB } & \text { NMDAR1 } \\ (120 K D)\end{array}$

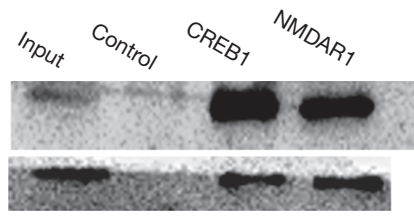

e

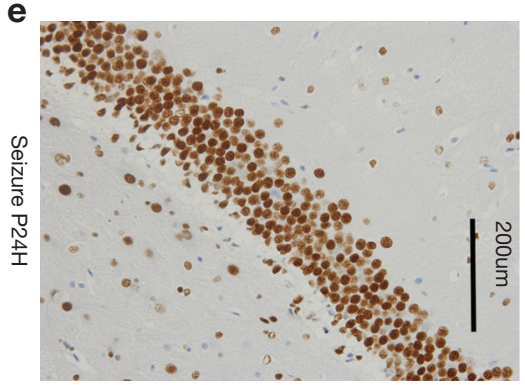

g
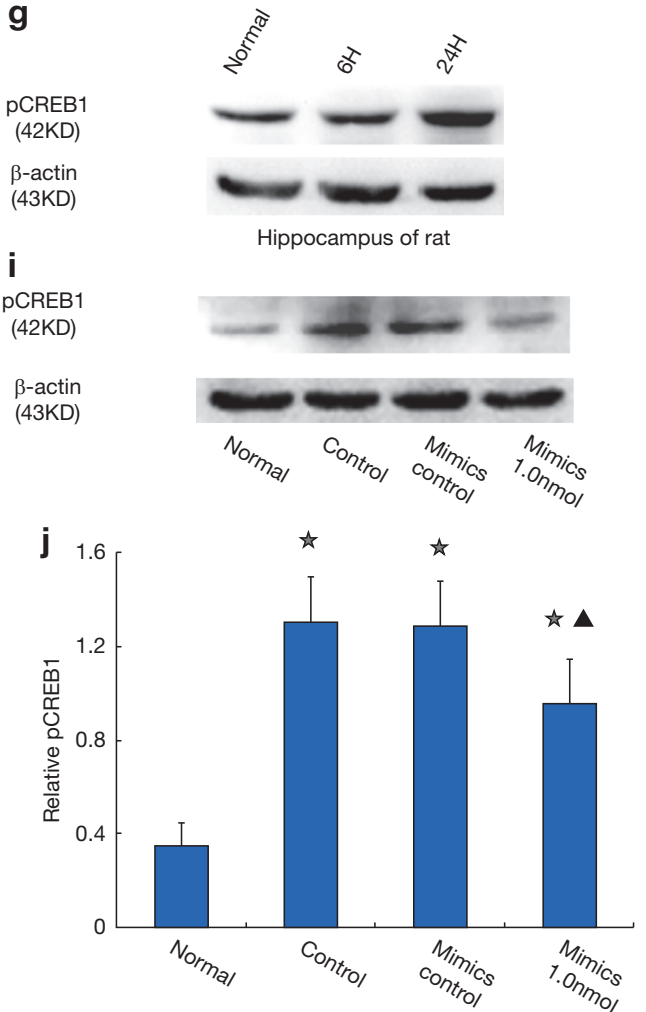

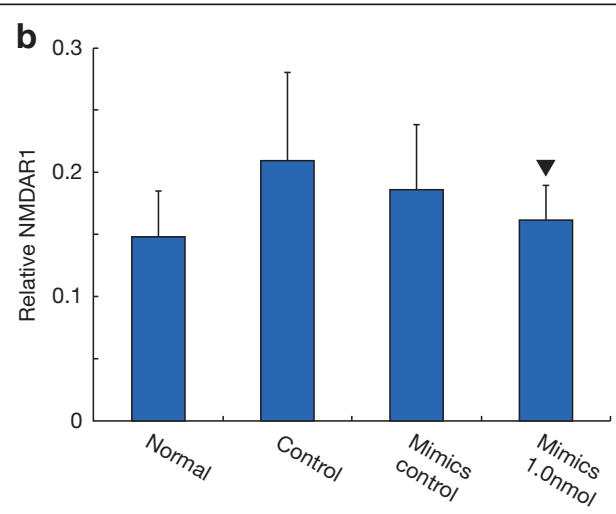

d

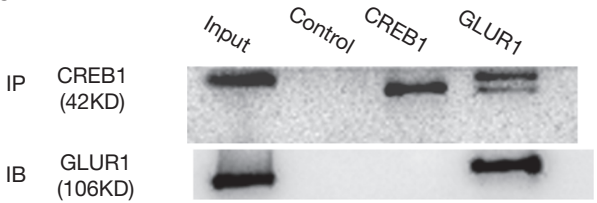

f
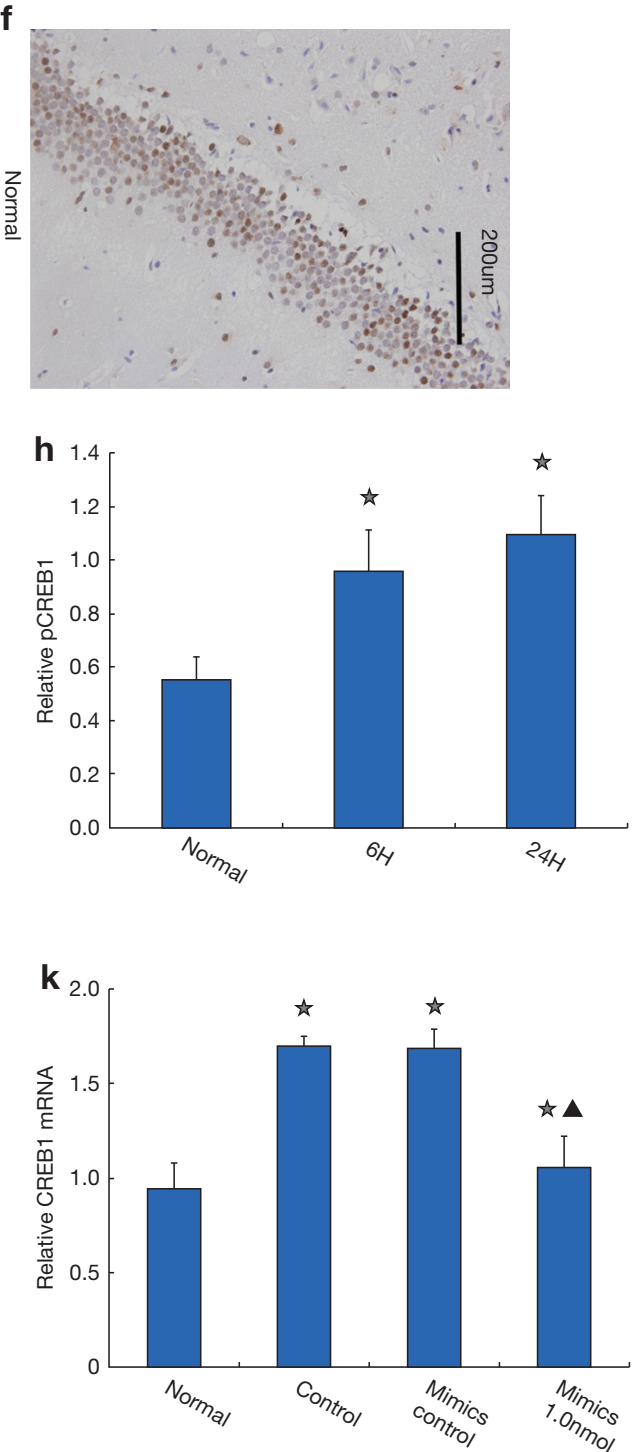

Effect of miR-124 on CREB1 activity in the hippocampus

Expert Reviews in Molecular Medicine (C) 2016 Cambridge University Press 
miR-124 downregulates AMPAR and NMDAR expression in the hippocampus of rat models

To test whether miR-124 alters AMPAR/NMDAR surface expression, we examined total and surface receptor expression by Western blot analysis. For AMPAR, a significant increase in the expression of both total and surface GLUR1 was present in the hippocampus of rats after pilocarpine-induced seizure activity (Fig. 5c and e). The scrambled mimics (agomir control) did not have an effect on AMPAR expression compared with the group with pilocarpineinduced seizure. The levels of total GLUR1 were significantly suppressed by miR-124, whereas the surface/total ratio of GLUR1 did not change significantly after microinjection of miR-124 mimics (Fig. 5c and e). For NMDAR, seizure activity led to a significant increase in NMDAR1 expression in the hippocampus. However, the surface and total expression of the NMDAR1 protein in the hippocampus of rats with pilocarpine-induced seizure were significantly lower in the miR-124 mimics group than they were in the mimics control group after $72 \mathrm{~h}$ of miR124 mimics injection or mimics control injection (Fig. 5d and f).

Subsequently, we studied whether preinjection of a miR-124 mimics affected the total expression of NMDAR after PTZ-induced seizures in rat models. After the injection of PTZ, seizure activity led to an increase in NMDAR1 expression in the hippocampus, and the miR-124 mimics partially decreased the expression of NMDAR1; however, none of these changes were significant ( $P>0.05$, Fig. $6 \mathrm{a}$ and b).

These results indicate that, in the hippocampus of rats, although the total expression of both NMDAR and AMPAR was suppressed by miR-124 $24 \mathrm{~h}$ after seizures were evoked, only the surface expression of NMDAR was downregulated by miR-124, suggesting that different mechanisms may be involved in the regulation of AMPAR and NMDAR trafficking.

Since there was significant change in AMPARmediated EPSCs, but there was no significant change in surface expression of GLUR1, we further examined whether the NMDAR1 and GLUR1 were correlated with acute increase of CREB1. Immunoprecipitation was used to test the interaction of CREB1 with NMDAR1 and GLUR1. Our studies confirmed that the CREB1 antibody effectively precipitated CREB1 and NMDAR1 but not GLUR1 from rat brain hippocampus (Fig. 6c and d).

\section{miR-124 inhibits CREB1 activity}

A previous study has demonstrated that miR-124 recognises specific binding sites in the $3^{\prime}$ UTR of the CREB1 mRNA in the neurons of Aplysia. The miR124-mediated CREB1 cascade contributes to enhanced synaptic plasticity (Refs 49, 50). In addition, the activation of CREB1 plays a key role in epileptogenesis (Refs 51, 52). Thus, we tested whether miR-124 inhibits neuronal hyperexcitability through CREB1. As shown in Figure 6, in the CA1 area, CA3 subfields, and dentate gyrus, CREB1 was expressed mainly in the nuclei of neurons. Immunostaining of activated CREB (Ser133-phosphorylated CREB, p-CREB) was significantly stronger in the hippocampus of rats with pilocarpine-induced seizure (Fig. 6e) $24 \mathrm{~h}$ after the first seizure compared with that observed in control rats (Fig. 6f). Consistently, Western blot analysis showed that p-CREB expression significantly enhanced 6 and $24 \mathrm{~h}$ after seizures in rats (Fig. $6 \mathrm{~g}$ and h). As shown in Figure 6i and j, hippocampal pCREB was significantly reduced after injection of miR-124 mimics. In addition, miR-124 significantly reduced CREB1 mRNA levels compared with control and scrambled miRNAs (Fig. 6k).

\section{Discussion}

The principal finding of this study was that miR-124 levels were suppressed in patients with TLE and in a rat model. Hippocampal injection of miR-124 mimics both alleviated seizure severity and prolonged the latency of seizure. MiR-124 administration inhibited neuronal firing, mEPSC and AMPAR- and NMDARmediated currents and NMDAR surface expression. The effects of pre-injection of miR-124 mimics on LFPs also showed shortened duration of epileptiformlike discharges and prolonged latency of epileptiformlike discharges in a model of pilocarpine-induced seizures. In addition, CREB1 activity was significantly down regulated by miR-124 in pilocarpine-induced seizures rats. We also demonstrated that CREB1 is a direct target of miR-124 in human HEK 293T cells. Furthermore, we showed that NMDAR-mediated current changes occur via direct interaction with

Effect of miR-124 on CREB1 activity in the hippocampus. (a) Sample Western blots of NMDAR1 in normal (without seizure inducing) and model rats (PTZ-induced seizure in rat models) without treatment (control), and in animals treated with mimics control and mimics. (b) Summary of the relative NMDAR1 analysed from h. $\nabla P>0.05$, compared with the normal, $n=4$. (c, d) Immunoprecipitation was used to survey the binding status between CREB1 and NMDAR1 or GLUR1. (e, f) Immunohistochemistry images show strong (e, 24 h after pilocarpine-induced seizures) and weak ( $\mathrm{f}$, control group) immunoreactive staining of pCREB in the dentate gyrus. Scale bar, 200 mm. (g) Sample Western blots of pCREB before (normal) and at different time points of pilocarpine-induced seizure. (h) Summary of pCREB activity showing a significant increase after seizures. ${ }^{*} P<0.05, n=5$. (i) Sample Western blots of pCREB in normal (without seizure inducing) and seizure rats without treatment (control), and in rats treated with mimics control and mimics. (j) Summary of the relative pCREB analysed from I. ${ }^{*} P<0.05$, compared with the normal. $\Delta P<0.05$, compared with the mimics control, $n=5$. (k) Relative CREB1 mRNA levels in normal (without seizure inducing) and seizure rats without treatment (control) and in animals treated with mimics control and mimics. ${ }^{*} P<$ 0.05 , compared with the normal. $\Delta P<0.05$, compared with the mimics control, $n=5$. 
CREB1. Our study revealed a previously unknown function of miR-124 in epilepsy.

The relationship between miRNA and epilepsy has received attention only recently. A genome-wide miRNA profiling study showed that, in human TLE, 165 mirRNAs were changed significantly, miR-124 being among those that were downregulated (Ref. 53). Consistently, our study demonstrated that decreased miR-124 expression occurs not only in patients with TLE, but also in animal models for up to 1 week. For the normal human hippocampal tissues as control were not available to study, we did not study the hippocampus in TLE patients. However, discrepancies exist regarding miR-124 changes in animal models. In a mouse model, miR124 was not included among the miRNAs that were up- or downregulated (Ref. 16). This may be owing to differences in animal species. Using a microarray method, $\mathrm{Hu}$ et al. investigated the miRNA expression profile in the same rat model as that used in our study, and found that miR-124a was significantly upregulated in these animals (Ref. 54). However, a quantitative PCR analysis was not performed to confirm this observation.

A limited number of studies have demonstrated that miR-124 may serve as a novel target for therapeutic intervention of neurological diseases. Intrathecal injection of miR-124 reduced persistent and neuropathic pain in a mouse model (Ref. 55). In EAE, peripheral administration of miR-124 leads to deactivation of macrophages and marked suppression of disease (Ref. 25). However, the current treatment for epilepsy is antisymptomatic. None of the antiepileptic drugs (AEDS) have been proven to be curative, and $20-30 \%$ of patients are refractory to AEDs (Refs 4, 56). In the present study, hippocampal injection of miR-124 caused a significant reduction in seizure severity in two rat models, indicating that miR-124 exerts a potential role in regulating neuronal excitability and seizure phenotype.

In the present study, miR-124 inhibited neuronal hyperexcitability by suppressing neuronal firing and mEPSC. In addition, NMDAR- and AMPAR-mediated currents and expression were also inhibited by miR124. Although it is relatively clear that miR-124 promotes embryonic neurogenesis and neuronal differentiation (Ref. 26), the function of miR-124 in the adult central nervous system and mature animals has not been well studied. In Aplysia, miR-124 suppresses serotonin-induced synaptic facilitation, thus providing direct evidence that miR-124 is critical in long-term synaptic plasticity in the mature nervous system (Ref. 49). A recent study in sensory neurons of Caenorhabditis elegans showed that miR-124 targets to genes that are associated with neurotransmitter release, cell-projection morphogenesis, and translation (Ref. 57), which supports a role for miR-124 in the regulation of synaptic plasticity in our experimental conditions.
It is well documented that CREB1 plays an important role in epilepsy. In patients with medically intractable epilepsy, enhanced CREB1 activation and gene expression occur in the seizure onset zone (Ref. 51). Activation of CREB1 and downstream genes contributes to epilepsy in both humans and rodent models (Ref. 52). Conversely, mice with decreased CREB1 levels exhibit a $\sim 50 \%$ reduction in spontaneous seizures, as well as a higher seizure threshold (Ref. 52). In our study, miR-124 administration resulted in CREB1 deactivation and mRNA suppression after epileptiform discharges, suggesting that CREB1 might partly mediate the miR-124 effect on synaptic remodelling. As reported previously, CREB1 enhances NMDAR but not AMPAR-mediated current and surface expression (Refs 58, 59). Consistently, NMDAR but not AMPAR trafficking to the cell surface was reduced, as measured by surface/total protein ratios in our study, suggesting a central role of CREB1 in neuronal excitability and seizure phenotype.

A previous study demonstrated that miR-124 recognised specific binding sites in the 3'UTR of the CREB1 mRNA in the neurons of Aplysia (Ref. 49). The miR124-mediated CREB1 cascade contributed to enhanced synaptic plasticity (Refs 49, 50). In our experiment, injection of miR-124 significantly reduced CREB1 mRNA, suggesting that CREB1 function is associated with miR-124. Using human HEK 293T cells, we have demonstrated that CREB1 is a direct target of miR-124 in human cells. Acute expression of active CREB1 correlates with an increase of both NMDAR surface expression and NMDAR-mediated currents. In this study, the altered activity and expression of CREB1 as a result of miR-124 administration, suggesting CREB1 is a potential mediator in miR-124 regulation of neuronal excitability, while NMDAR1 was a critical substrate of CREB1 which mediated the changes of NMDAR EPSCs.

The present study provided direct evidence that miR124 is inhibited in patients with epilepsy and in druginduced seizure rat models, and that the administration of miR-124 alleviates seizure attacks, which may lead to a novel intervention strategy for human epilepsy. However, the detailed mechanisms underlying miR124 regulation of CREB1 and neuronal excitability remain to be elucidated in the future.

\section{Acknowledgements}

This work was supported by the National Natural Science Foundation of China (Grant numbers 81271445 and 81501122) and the National Clinical Key Specialty Construction Foundation of China. The authors appreciate the patients and their families for their participation in this study. We sincerely thank the Tiantan Hospital and the Xuanwu Hospital of the Capital Medical University and the Xinqiao Hospital of the Third Military Medical University for providing the brain tissues, and the local 
ethics committee and the National Institutes of Health of China for their support.

\section{References}

1. McNamara J.O. (1999) Emerging insights into the genesis of epilepsy. Nature 399, A15-A22

2. McCormick D.A. and Contreras D. (2001) On the cellular and network bases of epileptic seizures. Annual Review of Physiology 63, 815-846

3. Rakhade S.N. and Jensen F.E. (2009) Epileptogenesis in the immature brain: emerging mechanisms. Nature Reviews Neurology 5, 380-391

4. McCorry D., Chadwick D. and Marson A. (2004) Current drug treatment of epilepsy in adults. The Lancet Neurology 3, 729-735

5. Simonato M. et al. (2012) Finding a better drug for epilepsy: preclinical screening strategies and experimental trial design. Epilepsia 53, 1860-1867

6. John B. et al. (2004) Human microRNA targets. PLoS Biology 2, e363

7. Bartel D.P. (2009) MicroRNAs: target recognition and regulatory functions. Cell 136, 215-233

8. Sayed D. and Abdellatif M. (2011) MicroRNAs in development and disease. Physiological Reviews 91, 827-887

9. Fineberg S.K., Kosik K.S. and Davidson B.L. (2009) MicroRNAs potentiate neural development. Neuron 64(3), 303-309

10. Bhalala O.G., Srikanth M. and Kessler J.A. (2013) The emerging roles of microRNAs in CNS injuries. Nature Reviews Neurology 9(6), 328-339

11. Jimenez-Mateos E.M. and Henshall D.C. (2013) Epilepsy and microRNA. Neuroscience 238, 218-229

12. Kosik K.S. (2006) The neuronal microRNA system. Nature Reviews Neuroscience 7, 911-920

13. Fiore R. and Schratt G. (2007) MicroRNAs in vertebrate synapse development. Scientific World Journal 7, 167-177

14. Schratt G. (2009) microRNAs at the synapse. Nature Reviews Neuroscience 10, 842-849

15. Nudelman A.S. et al. (2010) Neuronal activity rapidly induces transcription of the CREB-regulated microRNA-132, in vivo. Hippocampus 20, 492-498

16. Jimenez-Mateos E.M. et al. (2011) miRNA Expression profile after status epilepticus and hippocampal neuroprotection by targeting miR-132. The American Journal of Pathology 179, 2519-2532

17. Henshall D.C. (2013) Antagomirs and microRNA in status epilepticus. Epilepsia 54 (Suppl 6), 17-19

18. Roshan R.S. et al. (2014) Brain-specific knockdown of miR-29 results in neuronal cell death and ataxia in mice. RNA 20, 12871297

19. Lagos-Quintana M. et al. (2002) Identification of tissue-specific microRNAs from mouse. Current Biology 12, 735-739

20. Deo M. et al. (2006) Detection of mammalian microRNA expression by in situ hybridization with RNA oligonucleotides. Developmental Dynamics 235, 2538-2548

21. Yu J.Y. et al. (2008) MicroRNA miR-124 regulates neurite outgrowth during neuronal differentiation. Experimental Cell Research 314, 2618-2633

22. Sun A.X., Crabtree G.R. and Yoo A.S. (2013) MicroRNAs: regulators of neuronal fate. Current Opinion in Cell Biology 25(2), 215-221

23. Silber J. et al. (2008) miR-124 and miR-137 inhibit proliferation of glioblastoma multiforme cells and induce differentiation of brain tumor stem cells. BMC Medicine 6, 14

24. Fowler A. et al. (2011) miR-124a is frequently down-regulated in glioblastoma and is involved in migration and invasion. European Journal of Cancer 47, 953-963

25. Ponomarev E.D. et al. (2010) MicroRNA-124 promotes microglia quiescence and suppresses EAE by deactivating macrophages via the C/EBP-[alpha]-PU. 1 pathway. Nature Medicine 17, 64-70

26. Sonntag K.C., Woo T.U.W. and Krichevsky A.M. (2011) Converging miRNA functions in diverse brain disorders: a case for miR-124 and miR-126. Experimental Neurology 235, 427-435
27. Honchar M.P., Olney J.W. and Sherman W.R. (1983) Systemic cholinergic agents induce seizures and brain damage in lithiumtreated rats. Science 220, 323-325

28. Cavalheiro E.A., Santos N.F. and Priel M.R. (1996) The pilocarpine model of epilepsy in mice. Epilepsia 37, 1015-1019

29. Racine R.J. (1972) Modification of seizure activity by electrical stimulation: II. Motor seizure. Electroencephalography and Clinical Neurophysiology 32, 281-294

30. Kanter-Schlifke I. et al. (2007) Brain area, age and viral vectorspecific glial cell-line-derived neurotrophic factor expression and transport in rat. Neuroreport 18, 845-850

31. Hou J. et al. (2011) Identification of miRNomes in human liver and hepatocellular carcinoma reveals miR-199a/b-3p as therapeutic target for hepatocellular carcinoma. Cancer Cell 19, $232-243$

32. Davis C.J. et al. (2011) MicroRNA 132 alters sleep and varies with time in brain. Journal of Applied Physiology 111, 665-72

33. Zhang R. et al. (2014) MicroRNA-377 inhibited proliferation and invasion of human glioblastoma cells by directly targeting specificity protein 1 . Neuro Oncology 16, 1510-1522

34. Schmoll H. et al. (2003) Kindling status in sprague-dawley rats induced by pentylenetetrazole: involvement of a critical development period. The American Journal of Pathology 162, 10271034

35. Fang M. et al. (2011) Increased expression of Sonic hedgehog in temporal lobe epileptic foci in humans and experimental rats. Neuroscience 182, 62-70

36. Gassmann M. et al. (2009) Quantifying Western blots: pitfalls of densitometry. Electrophoresis 30, 1845-1855

37. Zhang Y. et al. (2015) Involvement of sigma-1 receptor in astrocyte activation induced by methamphetamine via up-regulation of its own expression. Journal of Neuroinflammation 17, 12, 29

38. Liu M. et al. (2011) miR-185 targets RhoA and Cdc42 expression and inhibits the proliferation potential of human colorectal cells. Cancer Letters 301, 151-160

39. Yin H. et al. (2011) Upregulation of liprin-al protein in the temporal neocortex of intractable epileptic patients and experimental rats. Synapse 65, 742-750

40. Wu P., Jiang L. and Chen H.S. (2010) Sodium valproate at the therapeutic concentration inhibits the induction but not the maintenance phase of long-term potentiation in rat hippocampal CA1 area. Biochemical and Biophysical Research Communications 391, 582-586

41. Zhong P. et al. (2003) Impaired modulation of GABAergic transmission by muscarinic receptors in a mouse transgenic model of Alzheimer's disease. Journal of Biological Chemistry 278, 26888-26896

42. Chen G. et al. (2006) Dopamine D3 receptors regulate GABAA receptor function through a phospho-dependent endocytosis mechanism in nucleus accumbens. The Journal of Neuroscience 26, 2513-2521

43. Gean P.W. and Shinnick-Gallagher P. (1988) Characterization of the epileptiform activity induced by magnesium-free solution in rat amygdala slices: an intracellular study. Experimental Neurology 101, 248-255

44. Jimenez-Mateos E.M. et al. (2012) Silencing microRNA-134 produces neuroprotective and prolonged seizuresuppressive effects. Nature Medicine 18, 1087-1094

45. Cao Q. et al. (2016) Elevated Expression of Acid-Sensing Ion Channel 3 Inhibits Epilepsy via Activation of Interneurons. Molecular Neurobiology 53, 485-498

46. Wang H. et al. (2015) MiR-124 regulates apoptosis and autophagy process in MPTP model of Parkinson's disease by targeting to Bim. Brain Pathology. doi: 10.1111/bpa.12267

47. Zhang H. et al. (2013) MIR-124 inhibits the migration and invasion of ovarian cancer cells by targeting SphK1. Journal of Ovarian Research 6, 84

48. An L. et al. (2013) microRNA-124 inhibits migration and invasion by down-regulating ROCK1 in glioma. PLoS One 8, e69478

49. Rajasethupathy P. et al. (2009) Characterization of small RNAs in Aplysia reveals a role for miR-124 in constraining synaptic plasticity through CREB. Neuron 63, 803-817

50. Preethi J. et al. (2012) Participation of microRNA 124-CREB pathway: a parallel memory enhancing mechanism of standardised extract of Bacopa monniera (BESEB CDRI-08). Neurochemical Research 37, 2167-2177 
51. Rakhade S.N. et al. (2005) A common pattern of persistent gene activation in human neocortical epileptic foci. Annals of Neurology 58, 736-747

52. Zhu X. et al. (2012) Decreased CREB levels suppress epilepsy. Neurobiology of Disease 45, 253-263

53. Kan A.A. et al. (2012) Genome-wide microRNA profiling of human temporal lobe epilepsy identifies modulators of the immune response. Cellular and Molecular Life Sciences 69, 3127-3145

54. Hu K. et al. (2011) Expression profile of microRNAs in rat hippocampus following lithium-pilocarpine-induced status epilepticus. Neuroscience Letters 488, 252-257

55. Willemen H. et al. (2012) MicroRNA-124 as a novel treatment for persistent hyperalgesia. Journal of Neuroinflammation 9 , 143

56. Brodie M.J. (2010) Antiepileptic drug therapy the story so far. Seizure 19, 650-655
57. Clark A.M. et al. (2010) The microRNA miR-124 controls gene expression in the sensory nervous system of Caenorhabditis elegans. Nucleic Acids Research 38, 3780-3793

58. Huang Y.H. et al. (2008) CREB modulates the functional output of nucleus accumbens neurons. Journal of Biological Chemistry 283, 2751-2760

59. Marie H. et al. (2005) Generation of silent synapses by acute in vivo expression of CaMKIV and CREB. Neuron 45, 741-752

${ }^{*}$ Corresponding author:

Jing Luo,

Department of Neurology, Chongqing Key Laboratory of Neurology,

The First Affiliated Hospital of Chongqing Medical University, 1 Youyi Road,

Chongqing 400016, China.

E-mail: jgire@163.com 